\title{
N-3 polyunsaturated fatty acid regulation of hepatic gene transcription
}

\author{
Donald B. Jump \\ Department of Nutrition and Exercise Sciences, Linus Pauling Institute, Oregon State University, \\ Corvallis, Oregon, USA
}

\begin{abstract}
Purpose of review-The liver plays a central role in whole body lipid metabolism and adapts rapidly to changes in dietary fat composition. This adaption involves changes in the expression of genes involved in glycolysis, de-novo lipogenesis, fatty acid elongation, desaturation and oxidation. This review brings together metabolic and molecular studies that help explain n-3 (omega-3) polyunsaturated fatty acid regulation of hepatic gene transcription.
\end{abstract}

Recent findings-Dietary n-3 polyunsaturated fatty acid regulates hepatic gene expression by targeting three major transcriptional regulatory networks: peroxisome proliferator-activated receptor $\alpha$, sterol regulatory element binding protein- 1 and the carbohydrate regulatory element binding protein/Max-like factor $\mathrm{X}$ heterodimer. $22: 6, \mathrm{n}-3$, the most prominent $\mathrm{n}-3$ polyunsaturated fatty acid in tissues, is a weak activator of peroxisome proliferator-activated receptor $\alpha$. Hepatic metabolism of $22: 6, \mathrm{n}-3$, however, generates $20: 5, \mathrm{n}-3$, a strong peroxisome proliferator-activated receptor $\alpha$ activator. In contrast to peroxisome proliferator-activated receptor $\alpha, 22: 6, \mathrm{n}-3$ is the most potent fatty acid regulator of hepatic sterol regulatory element binding protein-1. $22: 6, \mathrm{n}-3$ suppresses sterol regulatory element binding protein-1 gene expression while enhancing degradation of nuclear sterol regulatory element binding protein-1 through $26 \mathrm{~S}$ proteasome and Erk1/2-dependent mechanisms. Both n-3 and n-6 polyunsaturated fatty acid suppress carbohydrate regulatory element binding protein and Max-like factor X nuclear abundance and interfere with glucose-regulated hepatic metabolism.

Summary-These studies have revealed unique mechanisms by which specific polyunsaturated fatty acids control peroxisome proliferator activated receptor $\alpha$, sterol regulatory element binding protein-1 and carbohydrate regulatory element binding protein/Max-like factor X function. As such, specific metabolic and signal transduction pathways contribute significantly to the fatty acid regulation of these transcription factors and their corresponding regulatory networks.

\section{Keywords}

carbohydrate regulatory element binding protein; hepatic fatty acid metabolism; Max-like factor X; peroxisome proliferator activated receptor; polyunsaturated fatty acid $\alpha$; sterol regulatory element binding protein-1

\section{Introduction}

The type and quantity of fat ingested regulates hepatic lipid composition and gene expression [1]. Key targets for this control include glycolysis, de-novo lipogenesis (DNL), fatty acid elongation, desaturation and oxidation (Fig. 1 and Table 1) [2,3]. Other targets of fatty acid

Correspondence to Donald B. Jump, PhD, Department of Nutrition and Exercise Sciences, 107A Milam Hall, Oregon State University, Corvallis, OR 97331-5109, USA, Tel: +1 541737 4007; fax: +1 541737 6914; Donald.Jump@ oregonstate.edu. 
control have been identified by microarray analyses $[4,5]$. Although dietary fat has effects on mRNA turnover and processing [6], this review will focus on mechanisms by which $n-3$ polyunsaturated fatty acids (PUFAs) regulate key transcription factors controlling hepatic carbohydrate and lipid metabolism. Of the three n-3 PUFAs commonly encountered in the diet, $\alpha$-linolenic acid (18:3,n-3) is a weak regulator of hepatic gene expression, while eicosapentaenoic acid $(20: 5, \mathrm{n}-3)$ and docosahexaenoic acid $(22: 6, n-3)$ are strong regulators of hepatic gene expression $[3,7]$.

\section{Overview of polyunsaturated fatty acid-regulated hepatic gene transcription}

Several transcription factors have been identified as prospective targets for fatty acid regulation, including nuclear receptors: peroxisome proliferator-activated receptor (PPAR) family $\alpha, \beta, \gamma 1$ and $\gamma 2$, retinoid X receptor (RXR) $\alpha$, liver X receptor (LXR) $\alpha$ and hepatic nuclear factor (HNF-4) $\alpha$ and $\gamma$; and basic helix-loop-helix leucine-zipper transcription factors (bHLH-LZ): sterol regulatory element binding protein-1 (SREBP-1), carbohydrate regulatory element binding protein (ChREBP) and Max-like factor X (MLX)] [1,8-11]. Two general mechanisms characterize fatty acid control of these transcription factors. Fatty acids bind to and control the activity of specific nuclear receptors. In this fashion, fatty acids act like hydrophobic hormones regulating nuclear receptor function. Nonesterified fatty acids bind to PPAR ( $\alpha, \beta, \gamma 1$ and $\gamma 2$ ), HNF-4 ( $\alpha$ and $\gamma$ ), RXR $\alpha$ and LXR $\alpha$. Of these, PPAR subtypes are well recognized as fatty acid-regulated transcription factors in vivo [1]. Fatty acids control the nuclear abundance of SREBP-1, NFKB, ChREBP and MLX [8-10]. The mechanisms controlling these transcription factors are less clear, but likely involve changes in phosphorylation status of the transcription factor, which in turn controls transcription factor nuclear abundance and activity.

PPAR $\alpha$, SREBP-1c and ChREBP/MLX regulate multiple pathways involved in hepatic carbohydrate and lipid metabolism (Fig. 1 and Table 1). PUFA activation of PPAR $\alpha$ enhances fatty acid oxidation, while PUFA suppression of SREBP-1 and ChREBP/MLX results in the inhibition of DNL and PUFA synthesis. As such, PUFAs promote a shift in metabolism toward fatty acid oxidation and away from fatty acid synthesis and storage. This shift in metabolism will alter hepatic VLDL composition, which in turn affects extrahepatic lipid composition (Fig. 1) $\left[2,9,10,12,13^{\circ}\right]$. PUFA effects on lipid synthesis are seen in liver, but not necessarily in other tissues, like brain $[14,15]$.

\section{Fatty acid regulation of hepatic PPARa}

PPARs bind saturated, monounstaurated and polyunsaturated fatty acids. In cells and in vivo, hepatic PPAR $\alpha$ responds to changes in both exogenous (dietary) fat and newly synthesized fat (de-novo lipogenesis; DNL) [1,16]. This response is marked by changes in the expression of multiple enzymes involved in fatty acyl CoA formation and hydrolysis, fatty acid elongation and desaturation, and fatty acid oxidation (Table 1) $[3,17,18]$. Intracellular fatty acid ligands for PPAR, that is, nonesterified fatty acids (NEFAs), are maintained at low levels representing under $0.1 \%$ of the total cellular lipid [7]. In primary hepatocytes certain fatty acids are more effective activators of PPAR $\alpha$ than others. Challenging cells with a fatty acid that is normally abundant in cells, such as $18: 1, \mathrm{n}-9$, has little effect on intracellular NEFA mass and does not induce PPAR $\alpha$ activity. In contrast, challenging cells with a fatty acid that is typically a minor component of the cell, such as $20: 5, \mathrm{n}-3$, not only perturbs the NEFA fraction but also significantly activates PPAR $\alpha$ and induces its target genes, for example cytochrome P450-4A (CYP4A) and cytosolic fatty acyl thioesterase-1 (CTE1) [3,7].

Rodents fed fish oil-containing diets (at $<20 \%$ calories as fat) have multiple hepatic genes induced through PPAR $\alpha$ (Table 1) [2,3,12]. Although fish oil contains nearly equivalent amounts of $20: 5, \mathrm{n}-3$ and $22: 6, \mathrm{n}-3$, liver, plasma and extrahepatic tissues accumulate 22 : 
6,n-3. Surprisingly, $22: 6, \mathrm{n}-3$ is a weak activator of hepatic PPAR $\alpha$. When hepatocytes are treated with $22: 6, \mathrm{n}-3$, a small fraction of $22: 6, \mathrm{n}-3$ is metabolized to $20: 5, \mathrm{n}-3$ through a pathway called retroconversion [7,19] (Fig. 2).

Retroconverted fatty acids accumulate as esterified and nonesterified lipids. The generation of a preferred PPAR $\alpha$ ligand, such as $20: 5, \mathrm{n}-3$, through retroconversion may be a mechanism to control hepatic 22-carbon PUFA content. Enzymes involved in PUFA synthesis (elongases, desaturases and peroxisomal $\beta$-oxidation) are regulated by dietary fat, PPAR $\alpha$ and SREBP-1 (Table 1) $[12,20]$. The expression of these enzymes is altered during postnatal development [2] and in chronic metabolic diseases like obesity and diabetes [2,12,21,22]. Since these enzymes affect cellular abundance of PPAR $\alpha$ ligands, an important unanswered question is whether changes in the activity of these enzymes plays any role in controlling PPAR $\alpha$ activity or the progression of chronic metabolic disease.

\section{Hepatic glycolysis and de-novo lipogenesis is regulated by dietary polyunsaturated fatty acids}

Glycolysis and DNL are metabolic pathways for glucose utilization and fuel storage in the form of glycogen and triglycerides. Key transcription factors controlling glycolysis and DNL include SREBP-1, ChREBP, MLX and LXR (Fig. 1 and Table 1) $\left[13^{\circ}, 23,24^{\circ}, 25\right]$. PUFAs suppress at least one glycolytic enzyme, that is, L-pyruvate kinase (L-PK), and several enzymes involved in DNL and monounsaturated fatty acid synthesis, including ATP citrate lyase (ACL), acetyl CoA carboxylase (ACC), fatty acid synthase (FAS), SCD1 and Elovl-6 [1,8]. PUFAs, however, have no effect on the expression of enzymes involved in bile acid synthesis (CYP7A) or cholesterol efflux (ABCA1, ABCG5, ABCG8). As such, PUFA does not interfere with LXR $(\alpha$ or $\beta$ )-regulated gene expression [3].

\section{ChREBP and MLX}

Glucose effects on hepatic carbohydrate and lipid metabolism are mediated by glucose control of insulin secretion from pancreatic $\beta$-cells and insulin-stimulated glucose metabolism [13 ${ }^{\circ}$. In a recent report, glucose was found to activate LXR $\alpha$ and $\beta$ [24. ChREBP promoter and induces ChREBP gene transcription and the accumulation of mRNA $_{\text {ChREBP }}\left[26^{\circ}\right]$. Although there is considerable pretranslational control of ChREBP, much of the acute control by glucose and PUFA involves posttranslational mechanisms regulating ChREBP nuclear abundance $\left[8,13^{\circ}, 27,28^{\circ}\right]$. Glucose treatment of primary hepatocytes induces the accumulation of ChREBP in hepatocyte nuclei where ChREBP binds to carbohydrate regulatory elements (ChoRE) in promoters of responsive genes, including L-PK, ACC, FAS, and SCD1 (Table 1). Binding of ChREBP to ChoRE requires MLX [29]. Neither insulin nor glucose regulates MLX nuclear content [27,29].

Hormones that promote the accumulation of cAMP (glucagon or epinephrine) or metabolic states that activate AMP-kinase (starvation) induce ChREBP phosphorylation; phosphoChREBP does not accumulate in nuclei or bind well to ChoRE $\left[13^{\circ}, 28^{\circ}\right]$. Thus, the expression of genes involved in hepatic glycolysis and DNL are suppressed. In the well fed state, dietary glucose is in excess; glucose metabolism through the pentose phosphate pathway increases xylulose-5-phosphate production, a protein phosphatases 2A (PP2A) activator. PP2A dephosphorylates ChREBP allowing ChREBP to accumulate in nuclei [30]. The influx of ChREBP into nuclei triggers formation of ChREBP/MLX heterodimers on the ChoRE, as well as increased histone $\mathrm{H} 3$ and $\mathrm{H} 4$ acetylation and recruitment of RNA polymerase II to promoters of glucose responsive genes $\left[8,13^{\circ}, 29\right]$.

Both n-3 and n-6 PUFA inhibit L-PK gene transcription by suppressing ChREBP $[8,9]$ and MLX [8] nuclear abundance. PUFAs achieve this control through posttranslational 
mechanisms; PUFAs have no effect on mRNAs encoding ChREBP or MLX [8,9]. Although some have reported that PUFAs increase AMPK $\alpha$ phosphorylation [31], others have found no evidence for PUFA regulation of AMPK phosphorylation status [8,9]. While PUFAs affect both ChREBP and MLX nuclear abundance, overexpressed MLX, but not over-expressed ChREBP, abrogates PUFA control of L-PK and FAS [8]. These studies provide a key missing link in our understanding of how PUFAs control glycolysis (L-pyruvate kinase) and DNL (ACL, ACC, FAS, SCD1 and Elovl6). Unfortunately, the molecular basis for PUFA control of ChREBP and MLX nuclear abundance remains unresolved.

\section{SREBP-1}

SREBP-1c is one of three bHLH-LZ transcription factors (SREBP-1a, SREBP-1c and SREBP-2) controlling hepatic and whole body cholesterol and fatty acid synthesis. SREBP-1 regulates multiple pathways for fatty acid synthesis (Table 1) and VLDL assembly [23], while SREBP-2 controls cholesterol synthesis and uptake [23]. Mechanisms controlling SREBP nuclear abundance play a major role in determining the impact of SREBP on gene transcription. These posttranslational mechanisms include proteolytic processing [32] and 26S proteasomal degradation [33]. All SREBPs are synthesized as precursors (pSREBP, approximately 125 $\mathrm{kDa}$ ) tethered to the endoplasmic reticulum. Precursor SREBPs are escorted from the endoplasmic reticulum to the Golgi by SREBP-cleavage activating protein for proteolytic processing. Site 1 and site 2 proteases in the Golgi cleave the SREBP precursor to generate mature SREBP [32]. Mature SREBP is transported to the nucleus as a dimer, via importin- $\beta$ [34] where SREBP dimers bind sterol regulatory elements in promoters of target genes. Once bound, SREBPs recruit co-activators to promoters and stimulate gene transcription [23].

While sterols (cholesterol) suppress nuclear SREBP-2 levels by controlling the proteolytic processing step [35,36], PUFAs utilize multiple mechanisms to suppress SREBP-1 nuclear abundance [1]. Oxysterols (LXR agonists) and insulin induce SREBP-1 nuclear abundance [25]. While PUFAs do not interfere with LXR-regulated gene expression in hepatocytes or in vivo [3], PUFAs interfere with insulin control of SREBP-1 (Table 2) [11]. Insulin induces SREBP1c gene transcription through phosphoinositol-3 kinase and Akt-dependent pathways [37]. PUFAs transiently suppress insulin-stimulated Akt phosphorylation. Yet, overexpressed constitutively active Akt fails to abrogate PUFA suppression of SREBP-1 nuclear abundance [11]. Insulin induces hepatic mRNA encoding both SREBP-1a and 1c [38]. Insig 1 and 2 are resident endoplasmic reticulum proteins involved in SREBP processing [32]. PUFAs, like insulin, suppress Insig-2 expression [11]. Insulin inhibits the 26S proteasomal degradation of nuclear SREBP-1 $[11,33]$. The PUFA 22 : 6,n-3, but not other PUFAs, lowers SREBP-1 nuclear content through a 26S proteasomedependent mechanism [11]. While n-3 and n-6 PUFAs control SREBP-1 function by regulating SREBP-1c gene transcription and mRNA $_{\text {SREBP-1 }}$ turnover, only $22: 6$, n-3 regulates SREBP-1 through a $26 \mathrm{~S}$ proteasome-dependent mechanism.

These posttranslational mechanisms play a major role in controlling SREBP-1 nuclear abundance in vivo, particularly during the early postnatal period $[11,39]$. The very low levels of hepatic nuclear SREBP-1 in suckling rat pups [39] correlate with low blood insulin levels [40]. Injection of 12-day-old rats with insulin induces a prompt, but transient, rise in nuclear SREBP-1. Co-injection with insulin and a 26S proteasome inhibitor sustains elevated SREBP-1 levels in hepatic nuclei of suckling animals (unpublished observation). In primary rat hepatocytes, removal of insulin leads to a decline in SREBP-1 nuclear content $\left(t_{1 / 2}\right.$ approximately $10 \mathrm{~h}$ ); inhibitors of $26 \mathrm{~S}$ proteasomal degradation block this decline [11]. Treatment of primary hepatocytes with $22: 6, \mathrm{n}-3$ accelerates the loss of nuclear SREBP-1 ( $t_{1 / 2}$ approximately $4 \mathrm{~h}$ ); $26 \mathrm{~S}$ proteasome inhibitors completely block the $22: 6$-mediated suppression of nuclear SREBP-1. Regulating the $26 \mathrm{~S}$ proteasomal degradation appears to be 
a major mechanism controlling SREBP-1 nuclear abundance during early postnatal development and in adult animals. In contrast to SREBP-1, the 26S proteasome is not involved in the PUFA regulation of ChREBP or MLX nuclear abundance (unpublished observation).

SREBPs are phosphoproteins and their phosphorylation status controls their capacity to transactivate genes. cAMP-activated protein kinase A phosphorylates SREBPs within the DNA binding domain inhibiting SREBP binding to sterol regulatory elements [41]. SREBPs are phosphorylated by active (dephosphorylated) Gsk3 [33,42]. Phosphorylation of SREBP promotes ubiquitin ligase $\mathrm{SCF}^{\mathrm{Fbw}} 7$ binding, $\mathrm{SREBP}$ ubiquitination and $26 \mathrm{~S}$ proteasome degradation [33]. Insulin inhibits Gsk3 activity by stimulating Gsk3 phosphorylation through Akt [43]. Our preliminary studies indicate that $22: 6, n-3$, like insulin, induces Gsk $3 \beta$ phosphorylation. If these studies are confirmed, Gsk3 may not be the mediator of $22: 6, n-3$ control of SREBP-1 proteasomal degradation.

SREBPs are targets of Erk1/2 phosphorylation; Erk-phosphorylation of SREBP-1 impairs its regulation of LDL-receptor expression [44]. Treatment of primary hepatocytes with $22: 6, \mathrm{n}-3$ [11] or feeding rats fish oil (unpublished observation) induces hepatic Erk1/2 phosphorylation. Erk1/2 is a target of insulin action [45]. Insulin typically induces a rapid, but transient, increase in Erk1/2 phosphorylation in primary hepatocytes. Inclusion of $22: 6, n-3$ does not impair the rapid insulin-stimulated increase in Erk1/2 phosphorylation. Instead, $22: 6, \mathrm{n}-3$ prolongs elevated levels of Erk1/2 phosphorylation (Table 2). MEK inhibitors rapidly attenuate both $22: 6$-induced Erk 1/2 phosphorylation and $22: 6, \mathrm{n}-3$ suppression of SREBP-1 nuclear content implicating the MEK/Erk pathway in controlling SREBP-1 nuclear abundance [11]. The connections between the Erk1/2 pathway, the 26S proteasome and $22: 6, n-3$ control of SREBP-1 nuclear abundance remains unresolved.

Despite the apparent interference of n-3 PUFA with some facets of insulin action, n-3 PUFA, and in particular $22: 6, n-3$, mimics many insulin actions (Table 2). Moreover, in-vivo studies have established that $n-3$ PUFAs do not promote insulin resistance [46,47]. The basis for the selective control of insulin-regulated pathways by n-3 PUFA requires more study.

\section{Conclusion}

The central role of the liver in whole body lipid metabolism places it in a critical position to affect body fat composition. Dietary fat alters hepatic carbohydrate and lipid metabolism through changes in gene expression (Fig. 1 and Table 1). Several metabolic pathways are controlled by the PUFA-regulated transcription factors, PPAR $\alpha$, SREBP-1, ChREBP and MLX. Identification of these transcription factors as targets of PUFA control was a major advance in our understanding of how dietary fat controlled gene expression. Interestingly, these transcription factors display a differential response to PUFA. $20: 5$,n-3, but not $22: 6, \mathrm{n}-3$, is a potent activator of PPAR $\alpha .22: 6, n-3$, but not $20: 5, n-3$, controls SREBP-1 nuclear abundance through 26S proteasome and Erk-dependent mechanisms. Nuclear abundance of the ChREBP/ MLX-heterodimer, however, appears equally responsive to a wide range of 18-22-carbon n-3 and n-6 PUFAs. The significance of these differences will be revealed through more studies. In this regard, more study is required to define the metabolic and molecular basis for PUFA control of ligand availability for PPAR as well as mechanisms controlling SREBP-1, ChREBP and MLX function. Once defined, these mechanisms may provide new insights into disorders of lipid metabolism associated with chronic metabolic diseases such as diabetes and obesity.

\section{Acknowledgments}

I would like to thank Dr Maret Traber, Dr Moises Torres-Gonzalez and Ms Sasmita Tripathy for critically reading the manuscript. This project was supported by the National Institutes of Health (DK43220). 


\section{References and recommended reading}

Papers of particular interest, published within the annual period of review, have been highlighted as:

- of special interest

•• of outstanding interest

Additional references related to this topic can also be found in the Current World Literature section in this issue (pp. 315-317).

1. Jump DB, Botolin D, Wang Y, et al. Fatty acid regulation of gene transcription. J Nutr 2005;135:25032506. [PubMed: 16251601]

2. Wang Y, Botolin D, Christian B, et al. Tissue-specific, nutritional and developmental regulation of rat fatty acid elongases. J Lipid Res 2005;46:706-715. [PubMed: 15654130]

3. Pawar A, Botolin D, Mangelsdorf DJ, Jump DB. The role of liver X receptor-alpha (LXR-alpha) in the fatty acid regulation of hepatic gene expression. J Biol Chem 2003;278:40736-40743. [PubMed: 12917410]

4. Berger A, Roberts MA, Hoff B. How dietary arachidonic- and docosahexaenoic acid rich oils differentially affect the murine hepatic transcriptome. Lipids Health Dis 2006;5:108.

5. Patsouris D, Reddy JK, Muller M, Kersten S. Peroxisome proliferator-activated receptor alpha mediates the effects of high-fat diet on hepatic gene expression. Endocrinology 2006;147:1508-1516. [PubMed: 16357043]

6. Salati LM, Amir-Ahmady B. Dietary regulation of expression of glucose-6-phosphate dehydrogenase. Annu Rev Nutr 2001;21:121-140. [PubMed: 11375432]

7. Pawar A, Jump DB. Unsaturated fatty acid regulation of peroxisome proliferator activated receptoralpha activity in primary rat hepatocytes. J Biol Chem 2003;278:35931-35939. [PubMed: 12853447]

8. Xu J, Christian B, Jump DB. Regulation of rat hepatic L-pyruvate kinase promoter composition and activity by glucose, N-3 PUFA and peroxisome proliferator activated receptor-alpha agonist. J Biol Chem 2006;281:18351-18362. [PubMed: 16644726]

9. Dentin R, Benhamed F, Pegorier JP, et al. Polyunsaturated fatty acids suppress glycolytic and lipogenic genes through the inhibition of ChREBP nuclear protein translocation. J Clin Invest 2005;115:28432854. [PubMed: 16184193]

10. Jump DB. Fatty acid regulation of gene transcription. Crit Rev Clin Lab Sci 2004;41:41-78. [PubMed: 15077723]

11. Botolin D, Wang Y, Christian B, Jump DB. Docosahexaneoic acid [22: 6, n-3] stimulates rat hepatic sterol regulatory element binding protein-1c (SREBP-1c) degradation by an Erk- and 26S proteasome-dependent pathway. J Lipid Res 2006;47:181-192. [PubMed: 16222032]

12. Wang Y, Botolin D, Xu J, et al. Regulation of hepatic fatty acid elongase and desaturase expression in diabetes and obesity. J Lipid Res 2006;47:2028-2041. [PubMed: 16790840]

13. Postic C, Dentin R, Denechaud PD, Girard J. ChREBP, a transcriptional 3 regulator of glucose and lipid metabolism. Annu Rev Nutr 2007;27:179-192. [PubMed: 17428181]This is an excent review on the role ChREBP plays in carbohydrate and lipid metabolism.

14. Igarashi M, DeMar JC Jr, Ma K, et al. Upregulated liver conversion of alpha-linolenic acid to docosahexaenoic acid in rats on a 15 week n-3 PUFA-deficient diet. J Lipid Res 2007;48:152-164. [PubMed: 17050905]

15. Igarashi M, Ma K, Chang L, et al. Dietary n-3 PUFA deprivation for 15 weeks upregulates elongase and desaturase expression in rat liver but not brain. J Lipid Res 2007;48:2463-2470. [PubMed: 17715424]

16. Chakravarthy MV, Pan Z, Zhu Y, et al. "New" hepatic fat activates PPARalpha to maintain glucose, lipid, and cholesterol homeostasis. Cell Metab 2005;1:309-322. [PubMed: 16054078]

17. Hunt MC, Alexson SE. The role Acyl-CoA thioesterases play in mediating intracellular lipid metabolism. Prog Lipid Res 2002;41:99-130. [PubMed: 11755680] 
18. Tong F, Black PN, Coleman RA, Dirusso CC. Fatty acid transport by vectorial acylation in mammals: Roles played by different isoforms of rat long-chain acyl-CoA synthetases. Arch Biochem Biophys 2006;447:46-52. [PubMed: 16466685]

19. Sprecher H. Metabolism of highly unsaturated n-3 and n-6 fatty acids. Biochim Biophys Acta 2000;1486:219-231. [PubMed: 10903473]

20. Montanaro MA, Bernasconi AM, Gonzalez MS, et al. Effects of fenofibrate and insulin on the biosynthesis of unsaturated fatty acids in streptozotocin diabetic rats. Prostaglandins Leukot Essent Fatty Acids 2005;73:369-378. [PubMed: 16099631]

21. Brenner RR. Hormonal modulation of delta 6 and delta5 desaturases: case of diabetes. Prostaglandins Leukot Essent Fatty Acids 2003;68:151-162. [PubMed: 12538079]

22. Brenner RR, Rimoldi OJ, Lombardo YB, et al. Desaturase activities in rat model of insulin resistance induced by a sucrose-rich diet. Lipids 2003;38:733-742. [PubMed: 14506836]

23. Horton JD, Goldstein JL, Brown MS. SREBPs: activators of the complete program of cholesterol and fatty acid synthesis in the liver. J Clin Invest 2002;109:1125-1131. [PubMed: 11994399]

24- Mitro N, Mak PA, Vargas L, et al. The nuclear receptor LXR is a glucose 3 sensor. Nature 2007;445:219-223. [PubMed: 17187055]LXRs are well established targets of oxysterols. In this report, the authors present evidence indicating that LXR is regulated by glucose.

25. Kalaany NY, Gauthier KC, Zavacki AM, et al. LXRs regulate the balance between fat storage and oxidation. Cell Metab 2005;1:231-244. [PubMed: 16054068]

26•. Cha JY, Repa JJ. The liver X receptor (LXR) and hepatic lipogenesis. The 3 carbohydrate-response element-binding protein (ChREBP) is a target gene of LXR. J Biol Chem 2007;282:743-751. [PubMed: 17107947]This report established ChREBP as a target for LXR control. As such,

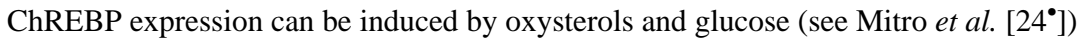

27. Towle HC. Glucose as a regulator of eukaryotic gene transcription. Trends Endocrinol Metab 2005;16:489-494. [PubMed: 16269245]

28•. Tsatsos NG, Davies MN, O'Callaghan BL, Towle HC. Identification and 3 function of phosphorylation in the glucose-regulated transcription factor ChREBP. Biochem J. 2008 Jan 9; [Epub ahead of print]. ChREBP function is regulated by its phosphorylation status. This report is the first to use a mass spectrometry approach to define phosphorylation sites in ChREBP.

29. Stoeckman AK, Ma L, Towle HC. Mlx is the functional heteromeric partner of the carbohydrate response element-binding protein in glucose regulation of lipogenic enzyme genes. J Biol Chem 2004;279:15662-15669. [PubMed: 14742444]

30. Kabashima T, Kawaguchi T, Wadzinski BE, Uyeda K. Xylulose 5-phosphate mediates glucoseinduced lipogenesis by xylulose 5-phosphate-activated protein phosphatase in rat liver. Proc Natl Acad Sci U S A 2003;100:5107- 5112. [PubMed: 12684532]

31. Suchankova G, Tekle M, Saha AK, et al. Dietary polyunsaturated fatty acids enhance hepatic AMPactivated protein kinase activity in rats. Biochem Biophys Res Commun 2005;326:851-858. [PubMed: 15607747]

32. Goldstein JL, DeBose-Boyd RA, Brown MS. Protein sensors for membrane sterols. Cell 2006;124:35-46. [PubMed: 16413480]

33. Sundqvist A, Bengoechea-Alonso MT, Ye X, et al. Control of lipid metabolism by phosphorylationdependent degradation of the SREBP family of transcription factors by SCGFBW7. Cell Metab 2005;1:379-391. [PubMed: 16054087]

34. Lee SJ, Sekimoto T, Yamashita E, et al. The structure of importin-beta bound to SREBP-2: nuclear import of a transcription factor. Science 2003;302:1571-1575. [PubMed: 14645851]

35. Adams CM, Reitz J, De Brabander JK, et al. Cholesterol and 25-hydroxycho-lesterol inhibit activation of SREBPs by different mechanisms, both involving SCAP and Insigs. J Biol Chem 2004;279:5277252780. [PubMed: 15452130]

36. Hirano Y, Yoshida M, Shimizu M, Sato R. Direct demonstration of rapid degradation of nuclear sterol regulatory element-binding proteins by the ubiquitin-proteasome pathway. J Biol Chem 2001;276:36431-36437. [PubMed: 11477106]

37. Ribaux PG, Iynedjian PB. Analysis of the role of protein kinase B (cAKT) in insulin-dependent induction of glucokinase and sterol regulatory element-binding protein 1 (SREBP1) mRNAs in hepatocytes. Biochem J 2003;376:697-705. [PubMed: 14505487] 
38. Xu J, Teran-Garcia M, Park JH, et al. Polyunsaturated fatty acids suppress hepatic sterol regulatory element-binding protein-1 expression by accelerating transcript decay. J Biol Chem 2001;276:98009807. [PubMed: 11124951]

39. Botolin D, Jump DB. Selective proteolytic processing of rat hepatic sterol regulatory element binding protein-1 (SREBP-1) and SREBP-2 during post-natal development. J Biol Chem 2003;278:6959_ 6962. [PubMed: 12488438]

40. Girard J, Ferre P, Pegorier J-P, Duee P-H. Adaptations of glucose and fatty acid metabolism during perinatal period and suckling-weaning transition. Physiol Rev 1992;72:507-562. [PubMed: 1557431]

41. Lu M, Shyy JY. Sterol regulatory element-binding protein 1 is negatively modulated by PKA phosphorylation. Am J Physiol Cell Physiol 2006;290:C1477-C1486. [PubMed: 16381800]

42. Punga T, Bengoechea-Alonso MT, Ericsson J. Phosphorylation and ubiquitination of the transcription factor sterol regulatory element-binding protein-1 in response to DNA binding. J Biol Chem 2006;281:25278-25286. [PubMed: 16825193]

43. Macaulay K, Doble BW, Patel S, et al. Glycogen synthase kinase 3alpha-specific regulation of murine hepatic glycogen metabolism. Cell Metab 2007;6:329-337. [PubMed: 17908561]

44. Roth G, Kotzka J, Kremer L, et al. MAP kinases Erk1/2 phosphorylate sterol regulatory elementbinding protein (SREBP)-1a at serine 117 in vitro. J Biol Chem 2000;275:33302-33307. [PubMed: 10915800]

45. Taniguchi CM, Emanuelli B, Kahn CR. Critical nodes in signalling pathways: insights into insulin action. Nat Rev Mol Cell Biol 2006;7:85-96. [PubMed: 16493415]

46. Perez-Matute P, Perez-Echarri N, Martinez JA, et al. Eicosapentaenoic acid actions on adiposity and insulin resistance in control and high-fat-fed rats: role of apoptosis, adiponectin and tumour necrosis factor-alpha. Br J Nutr 2007;97:389-398. [PubMed: 17298710]

47. Winzell MS, Pacini G, Ahren B. Insulin secretion after dietary supplementation with conjugated linoleic acids and n-3 polyunsaturated fatty acids in normal and insulin-resistant mice. Am J Physiol Endocrinol Metab 2006;290:E347-E354. [PubMed: 16188912] 


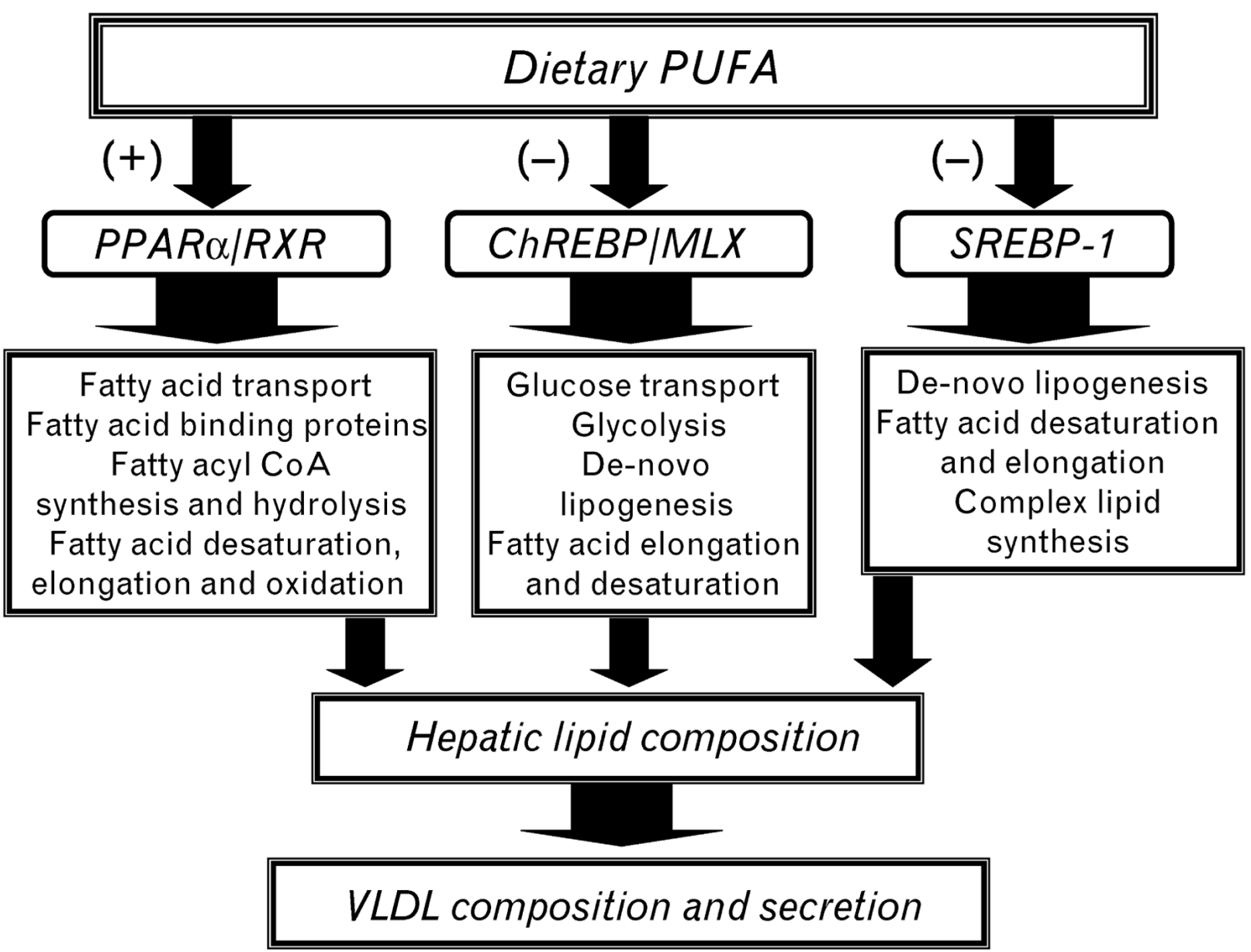

Figure 1. Impact of dietary polyunsaturated fatty acids (PUFAs) on hepatic gene transcription and lipid metabolism

Dietary polyunsaturated fatty acids (PUFAs) regulate three major transcriptional regulatory networks controlling multiple pathways involved in hepatic carbohydrate and lipid metabolism. PUFAs activate $(+)$ peroxisome proliferator-activated receptor (PPAR) $\alpha /$ retinoid $\mathrm{X}$ receptor (RXR) and suppress (-) the nuclear abundance of carbohydrate regulatory element binding protein (ChREBP)/Max-like factor X (MLX) and sterol regulatory element binding protein (SREBP-1). Changes in hepatic lipid composition affect VLDL composition. 


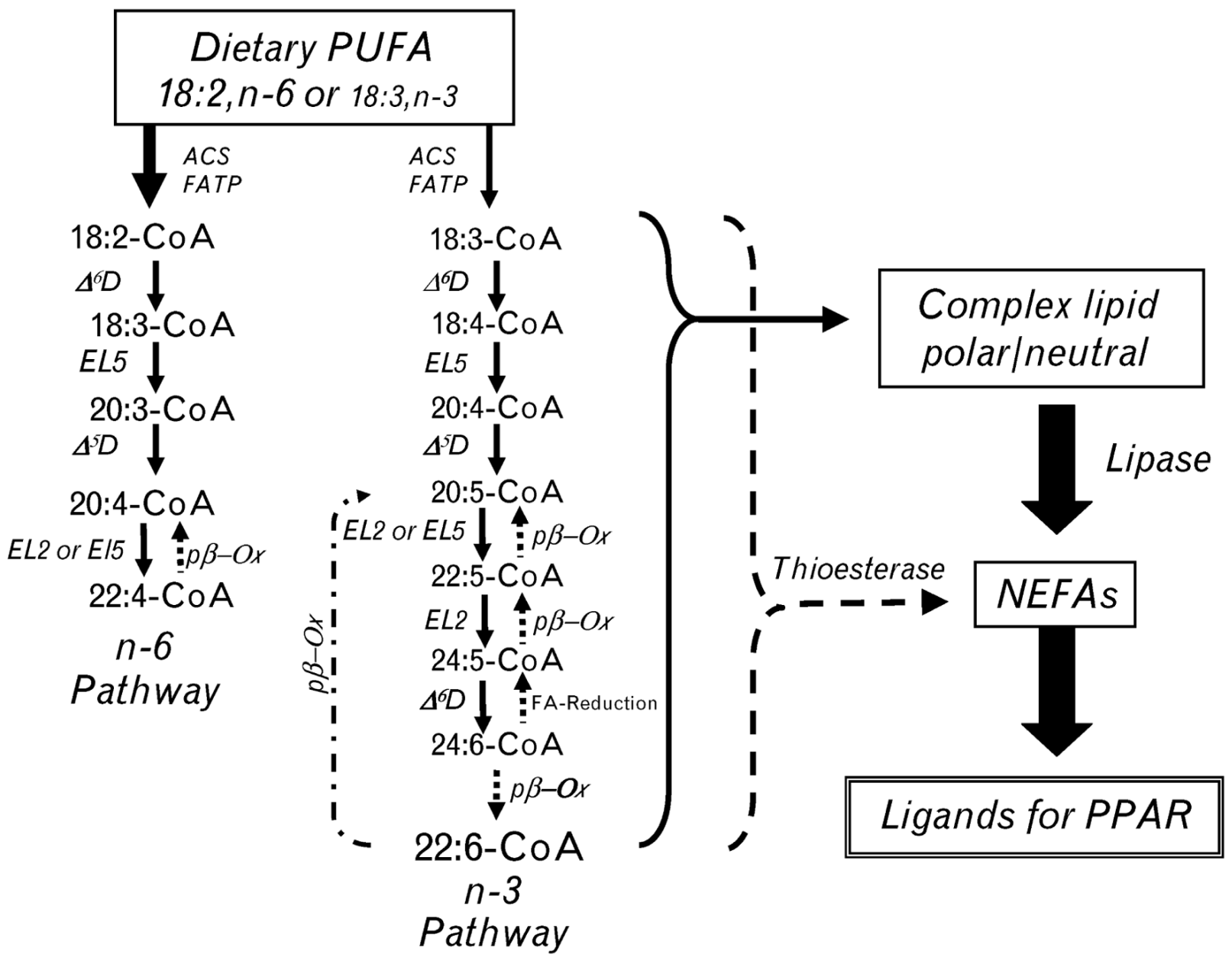

Figure 2. Metabolic pathways for n-3 and n-6 polyunsaturated fatty acid synthesis

The metabolic pathway for n-3 and n-6 polyunsaturated fatty acid (PUFA) synthesis (solid line/arrows), retroconversion of $22: 6, n-3$ to $20: 5, n-3$ (stippled line/arrows) and the release of fatty acids from fatty acyl coenzyme A (CoA) or complex lipids by the action of fatty acyl thioesterases (dotted line/arrow) or lipases (solid line, arrow), respectively. $18: 2, \mathrm{n}-6,20$ : 4,n-6 and $22: 6, n-3$ are the predominant PUFAs accumulating in tissues as neutral and phospholipids. ACS, acyl CoA synthetase; FATP, fatty acid transport protein; $\Delta^{5} \mathrm{D}, \Delta^{5}$-desaturase; $\Delta^{6} \mathrm{D}, \Delta^{6}$-desaturase; EL2, fatty acid elongase-2; EL5, fatty acid elongase-5; p $\beta$-Ox, peroxisomal $\beta$-oxidation; NEFAs, nonesterified fatty acids; PPAR; peroxisome proliferatoractivated receptor. 
Table 1

Transcription factors controlling hepatic glycolysis, fatty acid synthesis and oxidation

\begin{tabular}{|c|c|c|c|}
\hline Protein & SREBP-1 & ChREBP/MLX & PPARa \\
\hline \multicolumn{4}{|l|}{ Glycolysis and gluconeogenesis } \\
\hline Glucose transporter-2 (Glut2) & & $(+)$ & \\
\hline L-Pyruvate kinase (L-PK) & & $(+)$ & $(-)$ \\
\hline Phosphoenolpyruvate carboxykinase (PepCk) & $(-)$ & & $(+)$ \\
\hline \multicolumn{4}{|l|}{ Fatty acyl synthetases and thioesterases } \\
\hline Fatty acyl synthetase 1 (ACS1) & & & $(+)$ \\
\hline Cytosolic fatty acyl thioesterase (CTEI) & & & $(+)$ \\
\hline Mitochondrial fatty acyl thioesterase (MTEI) & & & $(+)$ \\
\hline Peroxisomal fatty acyl thioesterase (PTEI) & & & $(+)$ \\
\hline \multicolumn{4}{|l|}{ Fatty acid synthesis, desaturation and elongation } \\
\hline ATP citrate lyase (ACL) & $(+)$ & $(+)$ & \\
\hline Acetyl CoA carboxylase (ACC) & $(+)$ & $(+)$ & \\
\hline Fatty acid synthase (FAS) & $(+)$ & $(+)$ & \\
\hline$\Delta^{5}$-desaturase $\left(\Delta^{5} \mathrm{D}\right)$ & $(+)$ & & $(+)$ \\
\hline$\Delta^{6}$-desaturase $\left(\Delta^{6} \mathrm{D}\right)$ & $(+)$ & & $(+)$ \\
\hline Stearoyl CoA desaturase $\left(\Delta^{9} \mathrm{D}\right)$ & $(+)$ & $(+)$ & $(+)$ \\
\hline Fatty acid elongase-5 (Elov15) & & & $(+)$ \\
\hline Fatty acid elongase-6 (Elov16) & $(+)$ & $(+)$ & $(+)$ \\
\hline \multicolumn{4}{|l|}{ Fatty acid oxidation } \\
\hline Mitochondrial HMG CoA synthase (mtHMGCoASyn) & & & $(+)$ \\
\hline Peroxisomal acyl CoA oxidase (AOX) & & & $(+)$ \\
\hline Microsomal cytochrome P450-4A (Cyp4A) & & & $(+)$ \\
\hline
\end{tabular}

(+), Transcription factor induces enzyme expression; (-), transcription factor represses enzyme expression. 
Table 2

Comparison of insulin and n-3 polyunsaturated fatty acid effects on hepatic glycolysis, fatty acid synthesis and signaling mechanisms

\begin{tabular}{lll}
\hline & Insulin & n-3 PUFA \\
\hline Genes & & No effect \\
Glucokinase & Increase & Decrease \\
L-Pyruvate kinase & Increase & No effect \\
Phosphoenolpyruvate carboxykinase & Decrease & Decrease \\
Insig-2 & Decrease & \\
Fatty acid synthesis & Increase & Decrease \\
De-novo lipogenesis & Increase & Decrease \\
PUFA synthesis & & Decrease \\
Transcription factors & Increase & Decrease \\
Nuclear SREBP-1 & Increase & \\
Nuclear ChREBP and MLX & & Decrease \\
Cell signaling pathways & Increase & No effect \\
Akt phosphorylation (Activates) & Decrease & Increase \\
AMPK phosphorylation (Activates) & Increase & Increase \\
Erk1/2 phosphorylation (Activates) & Increase & \\
\hline
\end{tabular}

ChREBP, carbohydrate regulatory element binding protein; MLX, Max-like factor X; PUFA, polyunsaturated fatty acid; SREBP-1, sterol regulatory element binding protein. 